Bond University

Research Repository

\title{
A systematic review of the biomechanical research methods used in strongman studies
}

Hindle, Benjamin R; Lorimer, Anna; Winwood, Paul; Keogh, Justin W L

Published in:

Sports Biomechanics

DOI:

10.1080/14763141.2019.1598480

Licence:

CC BY-NC-ND

Link to output in Bond University research repository.

Recommended citation(APA):

Hindle, B. R., Lorimer, A., Winwood, P., \& Keogh, J. W. L. (2020). A systematic review of the biomechanical research methods used in strongman studies. Sports Biomechanics, 19(1), 90-119.

https://doi.org/10.1080/14763141.2019.1598480

\section{General rights}

Copyright and moral rights for the publications made accessible in the public portal are retained by the authors and/or other copyright owners and it is a condition of accessing publications that users recognise and abide by the legal requirements associated with these rights.

For more information, or if you believe that this document breaches copyright, please contact the Bond University research repository coordinator. 


\section{A Systematic Review of the Biomechanical Research Methods used in Strongman Studies}

Benjamin R. Hindle ${ }^{\mathrm{a}^{*}}$, Anna Lorimer ${ }^{\mathrm{a}, \mathrm{b}}$, Paul Winwood ${ }^{\mathrm{b}, \mathrm{c}}$ and Justin W. L. $\mathrm{Keogh}^{\mathrm{a}, \mathrm{b}, \mathrm{d}}$

${ }^{a}$ Faculty of Health Sciences and Medicine, Bond University, Gold Coast, Australia; ${ }^{b}$ AUT University, Sports Performance Research Institute New Zealand (SPRINZ), AUT Millennium Institute, Auckland, New Zealand; ${ }^{c}$ Faculty of Community Wellbeing and Development, Toi Ohomai Institute of Technology, Tauranga, New Zealand; ${ }^{d}$ University of the Sunshine Coast, Cluster for Health Improvement, Faculty of Science, Health, Education and Engineering, Sunshine Coast, Australia

Tel: +61 434889190

Email: bhindle@bond.edu.au

ORCID ID: 0000-0001-9601-9985

Co-Author email addresses:

alorimer@bond.edu.au

paul.winwood@toiohomai.ac.nz

jkeogh@bond.edu.au 


\section{A Systematic Review of the Biomechanical Research Methods used in \\ 2 Strongman Studies}

\section{Abstract}

4 As the sport of strongman is becoming increasingly popular, and such exercises are being

5 commonly used by strength and conditioning coaches for a wide range of athletic groups, a

6 greater understanding of the biomechanics of strongman exercises is warranted. To improve

7 the quality of research, this systematic review summarised the research methodology used in

8 biomechanical studies of strongman exercises and identified potential improvements to current

9 approaches. A search of five databases found ten articles adherent to the predefined inclusion

10 criteria. The studies assessed eight strongman exercises and included male participants of 11 relatively similar body mass but varying training backgrounds. Due to the complexity of 12 strongman exercises and the challenges in collecting advanced biomechanical data in the field, most studies used simplified measurement/analysis methods (e.g. 2D motion capture). Future strongman biomechanical studies should: assess under/un-researched strongman exercises; include a greater number of experienced and female strongman athletes; utilise more advanced (e.g. 3D motion capture and/or inertial sensor) technology so to provide a broader range and

17 greater quality of data. Such approaches will provide strength and conditioning coaches, strongman coaches and athletes with a greater understanding of strongman exercises, thereby further improving exercise prescription, athlete performance and minimising risk of injury.

Key words: Kinematics, kinetics, motion analysis, weightlifting 
Strongman is a competitive strength based sport consisting of exercises which are typically heavier versions of common activities of daily living, traditional tests of strength or more awkward/challenging variations of traditional weight training exercises such as the squat, deadlift and clean and press (Harris et al., 2016). Common strongman exercises utilise equipment such as; stones and loaded frames for lifting and carrying, logs and oversized dumbbells for overhead pressing, tyres for flipping and vehicles and loaded sleds for pulling (Keogh \& Winwood, 2017).

With the increasing popularity of strongman as both a competitive sport and as a source of alternative strength and conditioning training exercises for athletes of wide sporting backgrounds, the quantity and quality of research on the sport of strongman is continuing to increase. This research has examined the training and tapering practices of strongman athletes (McManus, O'Driscoll, Coleman, \& Wiles, 2016a; McManus, Wiles, Coleman, \& O'Driscoll, 2016b; Waller, Piper, \& Townsend, 2003; Winwood et al., 2018; Winwood, Keogh, \& Harris, 2011; Zemke \& Wright, 2011), how strength and conditioning coaches utilise strongman implements in their athletes' programmes (Winwood, Cronin, Keogh, Dudson, \& Gill, 2014b), the physiological responses to strongman training (Berning, Adams, Climstein, \& Stamford, 2007; Gaviglio, Osborne, Kelly, Kilduff, \& Cook, 2015; Ghigiarelli, Sell, Raddock, \& Taveras, 2013; Harris, et al., 2016; Winwood et al., 2015c; Woulfe, Harris, Keogh, \& Wood, 2014) and the injury epidemiology of strongman athletes (Winwood, Hume, Cronin, \& Keogh, 2014c). It should be acknowledged that some of this literature includes narrative reviews and/or opinion pieces on how strongman exercises could be best integrated into strength and conditioning programmes for non-strongman athletes.

Due to the emergent nature of the sport, wide range of exercises that may be performed in 
competition or training, and the apparent complexity of strongman exercises, it is expected that

47 some level of between study variation may be encountered when attempting to biomechanically analyse strongman exercises. Therefore, the primary objective of this systematic review was to collect and assess information on the research methods used in existing studies which primarily

50 focus on the biomechanical analysis of a strongman exercise. By addressing this primary 51 objective, the current systematic review will summarise the; exercises, study designs, study populations, and biomechanical analysis methods and measurements utilised in the existing literature. The secondary objective of this systematic review is to identify the gaps in the

54 research methodology used in strongman biomechanical studies. By identifying these major gaps, suggested improvements may be made to the current research methodology, better equipping future researchers with the knowledge required to conduct more comprehensive studies of greater quality on this sport. Such an approach will produce research which provides greater insight into how strongman exercises may be used in wider strength and conditioning or injury rehabilitation practice, as well as identify key biomechanical performance

60 determinants of these exercises for strongman athletes and coaches. 
Methods:

64 A review protocol for this paper was developed using the 'Preferred Reporting Items for Systematic Reviews and Meta-Analyses' (PRISMA) guidelines on reporting items for a systematic review and the associated PRISMA checklist (Shamseer et al., 2015). This was used in the planning and development of the systematic review to assure the quality of the review process.

69

Search Strategy and Inclusion Criteria:

An initial search was conducted using AusportMed, CINAHL, Embase, Medline (Ovid) and SPORTDiscus up to and including 2 July 2018. Due to the lapse in time between the initial search and submission for publication, a second search was conducted up to and including 25 October 2018. As the primary objective of this systematic review was to identify all strongman articles in which biomechanical analyses were performed, a two-level keyword search using Boolean operators was conducted. The first level of the search used terms associated with strongman exercises, lifts and training methods, while the second level of the search used terms associated with general biomechanical parameters. The full search strategy used for Medline (Ovid) was: (strongman OR strong man.tw OR strong-man.tw OR junkyard OR junk-yard OR junk yard OR log-lift* OR log lift* OR log press* OR log-press* OR yoke-walk OR yoke walk OR yoke-carry OR yoke carry OR super yoke OR super-yoke OR frame lift* OR frame-lift* OR frame carry OR frame-carry OR farmers walk OR farmers carry OR farmer's walk OR farmer's carry OR suitcase carry OR duck walk OR frame carry OR hercules hold OR husafell stone OR tyre flip* OR tyre-flip* OR tyre lift* OR tyre-lift* OR tire-flip* OR tire flip* OR tire lift* OR tire-lift* OR car flip* OR car-flip* OR atlas ston* OR stone lift* OR conans wheel OR conan's wheel OR fingal's fingers OR fingals fingers OR vehicle pull* OR vehicle-pull* OR sled pull* OR sled-pull* OR sled tow* OR sled-tow* OR truck pull* OR truck-pull* OR 
car pull* OR car-pull* OR chain drag* OR chain-drag* OR rope drag* OR rope-drag* OR sand bag* OR sand-bag* OR sandbag* OR car lift* OR car-lift* OR vehicle lift* OR vehiclelift* OR truck lift* OR truck-lift* OR arm over arm pull OR arm-over-arm OR keg toss OR keg-toss OR axle press* OR axle-press* OR dumbbell press* OR dumbbell-press*) AND (biomechanic* OR bio-mechanic* OR kinetic* OR kinematic* OR anthropomet* OR emg OR electromyograph* OR imu OR inertial measurement unit OR exp gait/ OR mechanic* OR force OR velocit* OR force-velocity OR time OR exp motion/ OR exp torque/ OR power OR body mass OR angular OR linear OR moment OR moment-angle OR moment angle OR momentarm OR moment arm OR momentum OR displac* OR equilibrium OR acceler* OR reac* OR joint OR pressure OR inertia* OR work OR energy OR potential OR injur* OR impuls* OR 3D OR motion capture).

In accordance with the intended exhaustive nature of the search strategy, no limitations were initially placed on language, year of publication, or literature source. To provide a systematic review that captures the methodology used when assessing complex strongman type exercises whilst being of value to the widest possible research community (and still adhering the topic of strongman and biomechanics), no restrictions were placed on the age, gender and lifting/athletic training experience of participants within a study. A set of guidelines outlining the inclusion and exclusion criteria was established by the author (Table 1).

107 Please insert Table 1 about here

All articles returned from the five searched databases were imported into online systematic review software Covidence (Veritas Health Innovation, Melbourne, Australia) and distributed to two independent reviewers. The software automatically removed duplicate

112 articles before each reviewer began the title and abstract screening process. Reviewers voted 
113 either 'yes', 'no' or 'maybe' to categorise each article's compliance with the pre-defined inclusion

114 criteria. Articles with vote combinations of 'yes'/'yes', 'yes/maybe' or 'maybe'/'maybe' were put

115 aside for full text screening while articles with vote combinations of 'no'/'no' were discarded

116 from further review. Articles not in the native language of the reviewers (English) were

117 returned by the respective database with sufficient translation for screening. Remaining articles

118 after title and abstract screening were then full text screened by reviewers with each reviewer 119 providing a reason for exclusion based on a hierarchical list of reasons. Where reviewers cast conflicting votes (such as 'yes'/'no' or 'maybe'/'no') during title and abstract screening or full text screening, or gave conflicting reasons for exclusion during full text screening, a consensus meeting was held to reach an agreement between both parties. A final scan of the reference list of all included articles was conducted to identify any relevant articles that were not initially found in the database searches. Forward citation tracking using Google Scholar was then employed to find any other articles that may have also been eligible to be included in the review.

Quality Assessment:

A risk of bias and quality assessment was undertaken by two independent reviewers. As no standard checklist appeared entirely suitable for the eligible cross-sectional biomechanical studies identified in this review, a checklist was developed by the authors based on systematic reviews including literature of similar study designs (Ariëns, van Mechelen, Bongers, Bouter, \& van der Wal, 2000; Davids \& Roman, 2014; DuRant, 1994; Gyorkos et al., 1994; Roman \&

134 Frantz, 2013; van der Windt et al., 2000; Vandenbroucke et al., 2007; Wong, Cheung, \& Hart, 135 2008). Where disagreements in the scoring was apparent between reviewers a consensus meeting was held to establish agreement. An item was scored as one where the article provided sufficient evidence in support of the criteria, and zero where the criteria was not met. A total 


\section{Results:}

150

risk of bias score was calculated for each article and categorised using the methods of Davids, et al. (2014), with articles scoring $\geq 67 \%$ considered as having a low risk of bias, articles scoring in the range of $34-66 \%$ considered as having a satisfactory risk of bias, and articles scoring $\leq 33 \%$ considered as having a high risk of bias. Only articles scoring a low or satisfactory risk of bias were included in the review.

\section{Data Analysis:}

To address the primary objectives of this systematic review, the data from the included articles were categorised into four main sections: Exercises/Objectives, Study Population, Study Design, and Biomechanical Analysis.

\section{Literature Search:}

The five databases originally searched on 2 July yielded 786 titles of which nine were found to be adherent to the inclusion criteria. After identifying another eligible study (Renals, Lake, Keogh, \& Austin, 2018) via a Google Scholar search, a second search of the five databases was performed so to include Renals, et al. (2018) (in press) in the review. The updated search on 25 October resulted in the addition of one article to the systematic review after the original search conducted on 2 July. A flowchart of the screening process undertaken on 25 October is presented in Figure 1.

Please insert Figure 1 about here

\section{Quality Assessment:}

Results from the risk of bias assessment are provided in Table 2. Generally, the articles 
reviewed provided a testable hypothesis, used well validated data collection methods, utilised appropriate statistical analysis methods, and presented results which were representative of the tests performed. After conducting the risk of bias assessment on the ten eligible articles, eight were assessed as having a low risk of bias $(\geq 67 \%)$, while two articles were assessed as having a satisfactory risk of bias (34-66\%).

Please insert Table 2 about here

\section{Exercises/Objectives:}

The ten eligible articles included in this systematic review have investigated eight different strongman exercises. Although some of the strongman exercises were assessed in multiple articles and some of the articles assessed multiple strongman exercises, the objectives, analysis methods and comparative measures seen in many of the articles exhibited some degree of between study variance.

\section{Exercises:}

The eight strongman exercises biomechanically analysed in the articles reviewed were the atlas stone lift, farmers walk, heavy sled pull, keg walk, log lift, suitcase carry, tyre flip and yoke walk (Figure 2).

Atlas stone lift: The atlas stone exercise requires the athlete to lift a large, spherical shaped stone off the ground and on to a chest height or higher ledge. In competition the exercise is usually performed as a series of stones of incremental mass which are lifted onto a series of different height ledges, with some competitions also involving the maximum number of repetitions within a minute performed with a stone of constant mass over a bar of constant height (McManus, et al., 2016b). 
Farmers walk: The farmers walk strongman exercise requires the athlete to pick up and move heavy objects carried in each hand. In competition the exercise is most commonly performed over a set distance of between 20 and $50 \mathrm{~m}$, with the athlete striving to complete the distance in the shortest possible time (Woulfe, et al., 2014).

Heavy sled/vehicle pull: The heavy sled/vehicle pull strongman exercise sees the athlete attached to a vehicle (or weight loaded sled) via a chest harness. The heavy sled pull variation is not often seen in competition, rather more commonly used as a training tool to simulate the competition vehicle pull. In both the heavy sled and vehicle pull, the athlete is most commonly required to pull the load a defined distance (often $20-25 \mathrm{~m}$ ) in the shortest possible time (McManus, et al., 2016b; Woulfe, et al., 2014).

Keg walk: The keg walk requires the athlete to carry a loaded keg on one of their shoulders. In this event, athletes are typically required to either transport a maximum number of kegs from one location to another in a defined period of time, or transport a defined number of kegs in the shortest possible time (Havelka, 2004).

Log lift: The log lift strongman exercise requires the athlete to lift a metal or wooden log from the ground and then push/press the implement above their head. In competition the exercise is either performed as a maximal load for a single repetition, or a submaximal load for a maximum number of repetitions in a defined period of time (often 60 seconds) (Havelka, 2004; McManus, et al., 2016b).

Suitcase carry: The suitcase carry requires the athlete to carry a loaded weight in one hand. In competition the exercise is typically performed for a defined distance in the shortest possible time (Havelka, 2004).

Tyre flip: The tyre flip strongman exercise requires the athlete to repeatedly flip a tractor tyre end over end. In competition this is typically performed over a defined distance, or for a defined 
number of repetitions in the shortest possible time (Keogh, Payne, Anderson, \& Atkins, 2010b;

213 McManus, et al., 2016b).

214 Yoke walk: The yoke walk requires the athlete to carry a loaded frame balanced across their 215 shoulders. In competition the exercise is either performed as a maximum distance in a defined 216 period of time, or a defined distance in the shortest possible time (Havelka, 2004).

218 Please insert Figure 2 about here

219

220

Objectives:

221 The earliest article on the biomechanics of strongman exercises was published by McGill, McDermott, and Fenwick (2009) and aimed to use biomechanical parameters to estimate back load, low-back stiffness and hip abduction torque when performing the atlas stone lift, farmers walk, keg walk, log lift, suitcase carry, tyre flip and yoke walk exercises. Keogh, et al. (2010b) used temporal measurements to determine possible factors which may affect athletic performance of the tyre flip exercise, while similar studies by Keogh and colleagues (Keogh et al., 2014; Keogh, Newlands, Blewett, Payne, \& Chun-Er, 2010a) used both temporal and kinematic measures to determine performance characteristics of the farmers walk and heavy sled pull exercises, respectively. Winwood, Keogh, and Harris (2012) sought to quantify the potential relationship between strength performance in weight training exercises and athlete anthropometrics, and strongman competition performance of various strongman exercises including the farmers walk, log lift, tyre flip and truck pull. A series of comparative studies published by Winwood and colleagues compared biomechanical measures of a variety of strongman exercises with those of technically similar traditional resistance training exercises (Winwood, Cronin, Brown, \& Keogh, 2014a, 2015a, 2015b). Most recently, Stastny et al. (2015) conducted a study to determine if muscle strength ratios could be used to predict muscle activation patterns during the farmers walk exercise, and Renals, et al. (2018) compared the 
effect of log diameter on force-time characteristics of the push press phase of the log lift.

\section{Study Population:}

241 The articles reviewed clearly detailed the number, age and body mass of participants included 242 in the study (Table 3). Although these variables exhibited some degree of variance between 243 studies, all studies consisted of male participants, with no studies including female participants.

244 Participants included in the articles reviewed typically had at least moderate levels of general 245 resistance training, one repetition maximum (1RM) testing or strongman type functional 246 training experience with many also having a combination of powerlifting and/or strongman competition experience.

Please insert Table 3 about here

250

Study Design:

252 All articles reviewed were of a cross-sectional observational study design. The general 253 structure of each study design consisted of a warm up protocol and a test protocol. The warm up protocol outlined in each study was of a general nature and inferred basic structural consistency for all participants. The test protocol of most studies detailed the number of sets and repetitions of a given exercise, the allocated rest period between sets/bouts of exercise and the prescribed implement load (Table 4).

The number of repetitions, sets and the way in which a set was defined varied between many of the articles reviewed. The variation in the definition of a set was generally seen in the studies whereby walking type strongman exercises were assessed. As strongman walking exercises such as the farmers walk, keg walk, heavy sled/vehicle pull, suitcase carry and yoke walk are typically performed once over a specific distance, the distance in which participants were required to perform these exercises during a trial varied between studies. Less variation 
was however seen in the definition of a set in the studies in which participants were required to perform repetitions of a static lift such as the log lift, stone lift and tyre flip. absolute implement load for all participants (Keogh, et al., 2014; Keogh, et al., 2010a; Keogh, et al., 2010b; McGill, et al., 2009; Winwood, et al., 2012), a set percentage of a participant's 1RM (Renals, et al., 2018; Winwood, et al., 2014a, 2015a, 2015b), or an incremental load based on the participant's six repetition maximum (6RM) (Stastny, et al., 2015). These loads were generally established in a familiarisation session held in the week/s prior to the testing session.

272

Please insert Table 4 about here

Biomechanical Analysis:

276 The reviewed articles analysed a variety of biomechanical parameters (Table 5) using a number of different measurement techniques and equipment. The biomechanical parameters seen in the articles reviewed have been categorised and presented for discussion using a deterministic model approach. The deterministic model is based on how the different categories of biomechanical measures may affect the ultimate performance outcome of the exercise (Figure $3)$.

Please insert Figure 3 about here

Temporal Measures:

286 Temporal data of the tyre flip (Keogh, et al., 2010b), farmers walk (Keogh, et al., 2014;

287 Winwood, et al., 2014a) and heavy sled pull (Keogh, et al., 2010a; Winwood, et al., 2015a) were collected using a series of cameras to capture two-dimensional (2D) data in the sagittal plane. Computer software was used to post process the video data and record the time taken 
for the athlete to complete each defined phase of the lift or section/phase of the walk/pull.

291 Temporal data for the fastest and slowest farmers walk, heavy sled pull and tyre flip trials were compared within and between participants in the studies by Keogh and colleagues (Keogh, et al., 2014; Keogh, et al., 2010a; Keogh, et al., 2010b), while Winwood, et al. (2014a) made group-average temporal data comparisons of the farmers walk to that of an unloaded walk, and Winwood, et al. (2015a) compared measures between phases of the heavy sled pull. Propulsion phase duration and total lift duration were measured for the log lift push press in Renals, et al. (2018), with such measures calculated from force plate data and compared between a barbell and various diameter logs.

299

Athlete/Implement Linear Kinematics:

Athlete linear kinematics were collected for the farmers walk (Keogh, et al., 2014; Winwood, et al., 2014a) and heavy sled pull (Keogh, et al., 2010a; Winwood, et al., 2015a) by method of marker based tracking using 2D sagittal plane video camera data and post processing computer software. This equipment and methodology was also commonly used to collect joint/segment angular kinematic data as described subsequently. The analysis performed on the athlete/implement kinematic measures for the farmers walk and heavy sled pull were as per the temporal measures presented previously for each respective study (Keogh, et al., 2014; Keogh, et al., 2010a; Winwood, et al., 2014a, 2015a).

Renals, et al. (2018) measured athlete linear kinematics during the log lift push press in

310 the form of vertical velocity and displacement of the athlete's centre of mass. These

311 measurements were calculated by subtracting the body mass of the athlete and the load lifted

312 from the vertical force data leaving the measurement of acceleration, which were then 313 integrated to give vertical velocity and integrated once again to give displacement. These measurements were presented as mean values during the braking and propulsive phases of the 
317 trajectory was plotted as vertical and horizontal displacement as both a function of time and

318 relative to the initial starting point, while velocity data were presented as peak and mean

319 vertical velocity values throughout each phase of the lift.

320

321

Joint/Segment Angular Kinematics:

322 Joint/segment angular kinematic data in the studies by Keogh and colleagues (Keogh, et al., 323 2014; Keogh, et al., 2010a), and Winwood and colleagues (Winwood, et al., 2014a, 2015a, 2015b) were collected using 2D video camera data techniques described in the athlete/implement linear kinematics section. The number of markers used to locate and track anatomical locations of the athlete's body ranged from six to 12 with anatomical positioning of

327 these markers varying depending on the exercise being analysed and the biomechanical parameter being assessed. These measures were presented as a range of motion throughout an exercise or an angle at defined instances throughout an exercise. consisted of a transmitter secured to the pelvis over the sacrum of the participant, and a receiver secured over the T12 spinous process of the participant, allowing for relative position of the lumbar spine to be approximated. In addition, a two video camera system that enabled vision of the frontal and sagittal plane was used to record and synchronise electromyography (EMG) data and spinal posture data obtained from the electromagnetic tracking system. These measures were presented as peak flexion-extension, medial/lateral bend, and twist of the lumbar spine. 
341 Kinetic measurements within the body of the athlete (in the form of muscle and joint loads)

342 and forces acting externally on the body (in the form of ground reaction forces) were reported

343 in five studies (McGill, et al., 2009; Renals, et al., 2018; Winwood, et al., 2014a, 2015a, 2015b).

344 Muscle and joint force, and torso stiffness estimations in McGill, et al. (2009) were derived by

345 first inputting the collected EMG data and spine angular kinematic data into a distribution 346 moment (DM) model (Ma \& Zahalak, 1991). Resultant muscle force and stiffness

347 approximations from the DM model along with spine angular kinematic data were then input

348 into a lumbar spine model based on anatomical approximations to optimise individual muscle

349 force and stiffness. The 18 degree of freedom model utilised an EMG based function to balance the external moment equation of a rigid link model (described subsequently) with the moments produced by the initial muscle and joint force estimations. This method ensured preservation of muscle recruitment patterns seen in the EMG data by adjusting individual muscle force and stiffness coefficients. Estimations of joint reaction moments about the lumbar spine (L4/L5) were derived in McGill, et al. (2009) through the input of digitised spine postural data and anthropometric approximations into a rigid link body model using similar techniques to McGill and Norman (1985). These moments were estimated for flexion/extension, medial/lateral bend and twist. Joint reaction moments of the hip were estimated by first recording a maximum voluntary

359 isometric hip abduction effort for each participant. Kinematic joint angle data in the frontal 360 plane from each of the walking exercises were digitised and input into the rigid link body model to estimate the hip abduction moment experienced throughout each exercise. These results were then normalised to the maximum isometric voluntary hip abduction produced by each participant and expressed as a percentage of the participant's maximum isometric voluntary hip abduction. 
USA) to collect ground reaction force data in the vertical, medial/lateral and anterior/posterior

367

directions (Winwood, et al., 2014a, 2015a, 2015b), while one study used a Kistler force plate (Model 9851B, Kistler Instruments Ltd., Hook, United Kingdom) to collect vertical ground reaction force data (Renals, et al., 2018). The data were post-processed using computer software and normalised for time, with forces presented in their respective axial directions depending on the exercise and study. Additionally, the log lift studies of Renals, et al. (2018) and Winwood, et al. (2015b) used the ground reaction force data and implement velocity data to estimate power and impulse throughout various phases of the lift.

\section{Muscular Activity:}

Electromyography measurements were collected in McGill, et al. (2009) using sixteen electrode pairs placed bilaterally on various abdominal, back and gluteal muscles. Standard EMG practices were generally reported throughout the preparation, collection and processing of the EMG data, with EMG signals full wave rectified and low-pass filtered using a secondorder Butterworth filter. These EMG signals were then normalised for each participant to a maximal voluntary contraction (MVC) of each muscle, providing insight into key muscular contributors during various strongman exercises. As detailed previously, these measurements were also used to calculate internal force and stiffness experienced by individual muscle fascicles of the lumbar spine during each exercise.

Stastny, et al. (2015) collected EMG data during the farmers walk exercise. A Noraxon Myosystem 1400A (Noraxon, Scottsdale, AZ, USA) EMG system was used to collect raw EMG data from four electrode pairs placed bilaterally on selected hamstring, quadricep and gluteal muscles. Standard EMG practices were generally reported throughout the preparation, collection and processing of the EMG data with data band-pass filtered and smoothed using a 
root mean square approach. Participants were required to perform MVC at $75^{\circ}$ knee

391 flexion/extension and $15^{\circ}$ hip abduction on an IsoMed 2000 Dynamometer (D \& R Ferstl

$392 \mathrm{GmbH}$, Hemau, Germany) prior to farmers walk testing to establish muscular strength ratios 393 of the hamstring/quadricep, hip abductor/quadricep, and hip abductor/hamstring. Participant

394 EMG data taken during the farmers walk trials were then normalised to MVC testing data and used to determine if a relationship could be established between lower limb muscle strength ratios and muscle activation patterns during the farmers walk.

397

398

Athlete Anthropometric Measures:

399

Athlete anthropometric measures of stature (height), body composition and body segment girths were taken in one of the articles reviewed (Winwood, et al., 2012). Stature measurements were taken using a portable stadiometer (Seca 214, Hangzhou, China), body segment girths

402 were taken using a Lufkin tape measure (Cleveland, OH, USA) and body composition 403 measurements were taken using a bioelectrical impedance machine (InBody230, Biospace, 404 Seoul, Korea). All anthropometric data were collected by a qualified International Society for the Advancement of Kinanthropometry anthropometrist, with the measurements used to determine if a relationship existed between athlete anthropometry and that of maximal strength 407 in traditional weight training exercises, and strongman exercise performance.

408

409 Please insert Table 5 about here

410

\section{Discussion and Implications:}

412 The methodology used to collect data for the biomechanical analysis of a movement may have

413 significant implications on the quality of the data and its applications to improving athletic 414 performance and/or reducing injury risk. The methodology selected by researchers may be 
415 influenced by the exercise being analysed, the objective of the study, study population, study

416 design and biomechanical measures desired, with each area discussed in order in the following

417 section. By exploring the methodologies used in biomechanical studies of traditional weight

418 training exercise, future biomechanical studies may produce a higher quality of data, which

419 should result in a more comprehensive understanding of this sport and therefore improve strongman performance and wider strength and conditioning practice.

421

422

\section{Exercises/Objectives:}

423 A large portion of the articles reviewed conducted biomechanical analysis on the farmers walk and heavy sled pull exercises (Keogh, et al., 2014; Keogh, et al., 2010a; McGill, et al., 2009; Stastny, et al., 2015; Winwood, et al., 2014a, 2015a; Winwood, et al., 2012). This is possibly due to the common occurrence of these exercises in the strength and conditioning programmes of non-strongman athletes (Winwood, et al., 2014a). Although biomechanically assessed in two of the ten articles reviewed, the heavy sled pull exercise is not typically seen in strongman competition, rather it is more commonly used as a training exercise for the vehicle/truck pull seen in competition. The heavy sled pull and the vehicle/truck pull may differ in terms of their performance determinants to some extent due to differences in the frictional behaviour of the two loads. To put a heavy sled in motion, static and dynamic sliding friction must be overcome, with typical coefficients of friction between a heavy sled and an athletic track found to range from 0.3 (static) to 0.47 (dynamic) (Cross et al., 2017). When compared to the coefficient of rolling resistance of a vehicle tyre $(\sim 0.004)$ (Hall \& Moreland, 2001), it may be appreciated that in order to overcome the initial inertia of an object of equal mass, a force 75 times greater must be applied to a sled (to overcome static sliding friction) than to a wheel (to overcome the friction apparent as rolling resistance). However, the mass and coefficient of friction are not the only variables that must be considered when assessing the replicability of a heavy sled pull 
440 to that of a vehicle pull. A phenomenon known as stick-slip must also be considered. This

441 phenomenon occurs as a result of an object in sliding contact generally having the inability to

442 momentarily continue to move once a propulsive force is no longer applied to the object as

443 would typically be seen with a wheel (Cross, et al., 2017). As a result of these behavioural

444 differences, the contribution of the current heavy sled pull studies toward improving

445 researcher's understanding of the key biomechanical determinants of the strongman

446 competition vehicle pull is still somewhat unclear.

Although the results from McGill, et al. (2009) made reference to some of the

biomechanical differences seen between athletes of varying competition standard, the results

449 were not statistically compared. The resultant back load, low-back stiffness and hip abduction torque measurements reported by McGill, et al. (2009) were compared between exercises. In a similar fashion, the biomechanical measurements taken in Renals, et al. (2018), and Winwood and colleagues (Winwood, et al., 2014a, 2015a, 2015b) were compared between strongman and traditional exercises, with no comparative analysis being undertaken between athletes of

454 varying performance levels. Stastny, et al. (2015) also did not measure or compare overall athlete performance, but rather compared muscular activation patterns between athletes of varying muscular strength ratios. The recommendations seen throughout these studies appear to be more directed at strength and conditioning coaches for targeted performance improvements in non-strongman athlete training programmes or for injury rehabilitation/prevention for both strongman and non-strongman athletes.

460 Contrary to McGill, et al. (2009), Renals, et al. (2018), Stastny, et al. (2015), and 461 Winwood and colleagues (Winwood, et al., 2014a, 2015a, 2015b), Keogh and colleagues 462 (Keogh, et al., 2014; Keogh, et al., 2010a; Keogh, et al., 2010b) compared biomechanical 463 measures between athletes of varying performance standards. Across these three studies it was 464 found that a number of biomechanical differences exist between athletes of varying 
465 performance levels which likely contribute to the overall performance of the athlete. The results 466 from Keogh and colleagues (Keogh, et al., 2014; Keogh, et al., 2010a; Keogh, et al., 2010b) 467 may be of particular value to strongman coaches and athletes wanting to improve competition 468 performance.

Future strongman studies should look to focus on popular strongman exercises with little to no previous research conducted in the field. Such exercises may include the atlas stone 471 lift, single arm dumbbell press, yoke walk and variations of the vehicle pull which are more representative of that seen in strongman competition. Additionally, future studies may consider comparing biomechanical measures between higher and lower performing athletes as has been

474 performed in few studies (Keogh, et al., 2014; Keogh, et al., 2010a; Keogh, et al., 2010b).

475 Identifying key biomechanical performance determinants of strongman exercises would be expected to improve coaching and the overall performance of strongman athletes at all levels

477 of competition. Information on how to better perform strongman exercises may also be used by special forces, police departments and emergency services personnel who are faced with a 479 life and death situation whereby they are required to move heavy, awkwardly shaped objects and/or carry or drag civilians to safety. Such tasks may be seen to closely replicate some of the exercises undertaken by strongman athletes (Keogh, et al., 2014).

\section{Study Population:}

484 The articles reviewed generally consisted of a small sample size (six or fewer participants). 485 Two of the articles reviewed included a larger number of non-strongman athletes $(n=16 ; n=$ 486 23) (Stastny, et al., 2015; Winwood, et al., 2012) with backgrounds in other forms of resistance training. Although results from these non-strongman populations may be of relevance to strength and conditioning coaches who are contemplating including strongman exercises into an athlete's training programme, the results from these studies may not be representative of, or 
generalisable to the competitive strongman athlete population. In addition, the inclusion of non-

491 strongman athletes in some of the studies reviewed likely had a small carry-over effect on subsequent methodology used in the study, such as the warm-up methods and the loads used when performing a given exercise. These considerations will be discussed further in subsequent sections. The small number of competitive strongman athletes included in the articles reviewed may be due to the sport of strongman still being young and the limited number of athletes competing in the sport of strongman in any given geographical location. With the increasing popularity of the sport of strongman it may be expected that future studies will include a greater sample size of national and international level competitive strongman athletes, including female and lighter male participants than have been included in previous studies. Studies of typical strongman athletes would provide results which are of greater relevance to strongman coaches and athletes.

502

503

\section{Study Design:}

504 All articles included in the review were of cross-sectional design. This type of study design is 505 commonly utilised in biomechanical research and provides a snapshot of athlete performance 506 and biomechanical parameters at a single point in time. These performance outcomes may be 507 affected by how an athlete is feeling on a particular day and may be influenced by factors such as sleep, stress, nutrition, training load, injury or illness. No articles published to date have assessed and/or compared biomechanical parameters of an athlete performing a strongman

510 exercise over an extended period of time. Future studies may consider assessing strongman

511 training performance and biomechanical parameters at regular intervals throughout the training and competition season of an athlete. The results from such studies may be of particular interest to strongman coaches when programming training blocks for athletes, determining associations

514 between strongman technique and performance, and also in assessing signs of adaptation, over- 
515 training, fatigue and injury. Strength and conditioning coaches may also be interested in such

516 longitudinal studies as they would provide greater insight into the benefits and potential injury

517 risks of such exercise programmes.

518 The way in which implement loading was determined in the articles reviewed exhibited

519 some degree of between study variation. The majority of loads used were somewhat reflective

520 of the experience and/or competitive standard of the athletes tested, with studies that included

521 a greater number of non-strongman athletes typically seeing lighter loading. Many of the

522 articles reviewed lacked detail on the methods used to establish implement load. These details,

523 along with justifications for the use of the method should be reported to provide the reader with

524 a greater context to the study. Prescribing implement loading based on pre-test 1RM tests could

525 be a useful approach in some studies, as it would provide a way to normalise the data collected

526 based on the participants' muscular strength. This approach has been used in four studies that

527 focused on a comparison of strongman and traditional lifts, with the results assisting in

528 improving the understanding of how strongman exercises may be best, if at all, included in the

529 strength and conditioning programmes of non-strongman athletes (Renals, et al., 2018;

530 Winwood, et al., 2014a, 2015a, 2015b). However, basing loads on an athlete's 1RM does not

531 mimic actual strongman competition, whereby athletes of a given gender and body mass

532 category compete with the same absolute loads for each exercise. Utilising such competition

533 loading approach in strongman performance research may be of major interest to strongman

534 coaches and athletes as it provides insight into the most important factors influencing

535 strongman competition performance.

536 It is also apparent that no strongman biomechanical study to date has assessed an

537 exercise over a range of loads as may be experienced during training and as is standard practice

538 for examining force-velocity-power relationships in traditional resistance training exercises

539 (Blatnik et al., 2014; Rahmani, Viale, Dalleau, \& Lacour, 2001; Sanchez-Medina, Gonzalez- 
540 Badillo, Perez, \& Pallares, 2013). Both strongman coaches and athletes, and strength and

541 conditioning coaches of non-strongman athletes would benefit from such analyses as it may

542 assist in the prescription of loading during a training session or phases of a periodisation

543 training programme where specific performance outcomes are desired.

544 Limited detail on the warm up protocol undertaken by participants was provided in

545 articles reviewed. Few articles explicitly stated whether an athlete self-directed or a warm up

546 routine developed by the researcher was used, making it difficult for the reader to determine

547 the suitability of the methods selected. It could be expected that altering the usual warm up

548 protocol of an experienced athlete by enforcing a researcher designed warm up routine may

549 affect the athlete's performance during testing. Future studies should provide greater detail on

550 the warm up protocol used, and where experienced strongman athletes are included should use

551 an athlete self-directed warm up routine, with all warm up elements documented by the supervising researchers. There is also no research that has quantified the effect different warm up approaches may have on strongman performance and biomechanics. Therefore, it would

554 also be useful for researchers interested in strongman performance to compare the effects of different warm up approaches (including the potential use of post-activation potentiation) on performance in simulated strongman competitions to determine what may constitute optimal warm up strategies for the sport.

558

\section{Biomechanical Analysis:}

560 The majority of the articles reviewed used 2D kinematic analysis to estimate sagittal plane 561 temporal measures, athlete linear kinematics and joint/segment angles. The reliance on 2D

562 kinematic analysis of these strongman exercises is a potential major limitation of this research, whereby three-dimensional (3D) motion capture is considered the gold standard of describing athlete and object kinematics. Escamilla et al. (2000) compared 2D versus 3D kinematic 
analysis for athletes performing the conventional and sumo deadlift. Greater differences

566

567

568

569

570

571

572

573

574

575

576

577

578

579

580

581

582

583

584

585

586

587

588

589

between joint/segment angles obtained using 2D versus 3D kinematic analyses were seen for the sumo deadlift than the conventional deadlift. The study suggested that these differences could be attributed to the multi-planar movement of the lower body in the sumo deadlift, which requires a wider stance and greater angle at which the feet are turned out compared to the conventional deadlift. Such results indicated that 2D kinematic analysis shows strong correlation with 3D kinematic analysis for knee, thigh and hip angular motion which is primarily performed in the sagittal plane only (such as the conventional deadlift). However, measurement errors are to be expected when performing 2D kinematic analysis on multi-planar movements in which some of the movement occurs at an angle that is not perpendicular to the field of view of the camera, as is often seen in many strongman exercises such as truck pull, tyre flip and weighted carries such as the farmers and yoke walk. Schurr, Marshall, Resch, and Saliba (2017) has also shown that 2D kinematic analysis is comparable to 3D motion capture when evaluating ankle, hip, knee and trunk angles in the sagittal plane during a single leg squat. There was however, no significant correlation between the two methods at any of the joints in the frontal plane except for a poor correlation at the knee. The discrepancies in the frontal plane were suggested to be attributed to the possible rotation of the ankle, hip and knee joints throughout the movement, as well as the high relative error of these joint motions that reflects the limited range of motion of these joints in the frontal compared to the sagittal plane.

Although McGill, et al. (2009) successfully collected 3D motion data of athletes performing a number of strongman exercises, the focus was on the lower back and required data collection equipment to be attached to the posterior of the body only. While the use of a similar gold standard approach such as 3D optical motion capture may provide a greater quality of biomechanical measurements, especially for multi-planar movements that are not perpendicular to the camera's field of view as occurs relatively often in a number of strongman 
exercises, several difficulties in the use of this method may be experienced when applied to strongman exercises. Three-dimensional optical motion capture typically requires the placement of around 50 markers on various anatomical locations and planes of body segments to capture accurate translational and rotational motion (Guerra-Filho, 2005). Strongman exercises often require large, heavy, and awkward to position/lift implements (such as logs and stones) to be lifted over large portions of the body's anterior surface, thus it would appear difficult to successfully secure reflective markers to the required anatomical locations of an athlete's body whilst ensuring the markers would not be obscured or displaced when performing these exercises.

Recent developments in inertial measurement unit (IMU) based motion capture systems may provide a more feasible means of collecting 3D data than traditional 3D optical motion capture techniques (Blair, Duthie, Robertson, Hopkins, \& Ball, 2018). Inertial measurement unit motion capture systems utilise a network of sensors located at various locations on the body, with the sensors secured either on the skin surface or on top of or beneath clothing. The

604 development of such systems has seen various methods used for calibration, thus the versatility 605 of locating the sensors on the body provides the potential to overcome issues seen when using 606 traditional 3D motion capture systems (Filippeschi et al., 2017). Future studies may consider

607 the use of IMU systems to improve the quality and breadth of motion data collected, with such an approach likely to be able to be utilised in both competition and training settings. Force-velocity-power profiles of the barbell or the combined body-barbell system are 610 becoming more commonly used to prescribe training load, and assess and/or predict the 611 performance of an athlete (Argus, Gill, Keogh, \& Hopkins, 2011; Giroux, Rabita, Chollet, \& 612 Guilhem, 2016; Pearson, Cronin, Hume, \& Slyfield, 2009; Picerno et al., 2016; Swinton, 613 Stewart, Agouris, Keogh, \& Lloyd, 2011). Two of the articles included in the current review 614 obtained measurements of mean implement velocities, and mean force and power production 
615 during the strongman log lift (Renals, et al., 2018; Winwood, et al., 2015b). Presenting these

616 measurements as a function of time or load may be of particular interest to strongman coaches

617 and athletes, and strength and conditioning coaches where force-velocity-power training

618 principles are considered. Future research may investigate the use of force-velocity-power

619 profiling as a tool for prescribing training strategies and predicting the success of a strongman

620 lift.

621

Standard EMG protocol procedures were generally followed in the two strongman

622

studies that assessed muscle activity, however the inherent challenges associated with using

623

EMG data to represent muscle activity must be acknowledged. These issues have generally

624 been attributed to the noise generated at the skin-electrode interface due to the relative movement between the electrode, skin and muscle, the noise generated by electromagnetic radiation from nearby electrical appliances, and internal cross talk detected from surrounding muscles (Chowdhury et al., 2013; Vigotsky, Halperin, Lehman, Trajano, \& Vieira, 2018). the EMG signal produced during MVC readings still most commonly utilised. There are however issues with normalising to MVC, especially when it is observed that one of the articles reviewed had muscle activity readings for several muscles greater than $100 \%$ of the athlete's pre-test MVC (McGill, et al., 2009). Ball and Scurr (2010) compared repeated (across multiple days and weeks) EMG activity measurements of the triceps surae muscles whilst performing a

634 variety of exercises including the squat jump, $20 \mathrm{~m}$ sprint, isometric heel raise and isokinetic 635 plantar flexion. It was theorised that these exercises may provide a means of EMG normalisation reference values for the triceps surae muscles. While EMG activity

637 measurements of all triceps surae muscles were reliable when performing the squat jump over 638 multiple days and weeks, measurements taken when performing the $20 \mathrm{~m}$ sprint, isometric heel 639 raise and isokinetic plantar flexion displayed less reliability, with reliability dependant on the 
640 duration between retests and the muscle being measured. Although the challenges associated

641 with EMG readings are often acknowledged by researchers and a number of techniques have

642 been developed to reduce the likelihood of misinterpretation of data (Chowdhury, et al., 2013),

643 there are currently few practical alternative methods of acquiring and normalising muscle

644 activity data.

645 For a body to displace a load, a muscular torque exceeding the load torque must be

646 produced. The muscular torque can be defined as the product of force produced by the muscles

647 spanning the joint and their respective muscular moment arm lengths, and the load torque can

648 be defined as the product of the load force and the load moment arm length. Thus, the limb

649 length and girth of the segment contribute substantially to the resistance moment arm length, muscular force produced and performance outcome of the exercise. Of the articles reviewed, only one study measured participant body composition and anthropometry, with the measurements obtained at one point of time from non-strongman (rugby) athletes who performed a range of strongman exercises (Winwood, et al., 2012). Although the study found large to very large correlations between overall strongman competition performance and many anthropometric measures, measurements of limb lengths were not included in the analysis, thus presenting a potential gap in the research methodology. utilised (Musser, Garhammer, Rozenek, Crussemeyer, \& Vargas, 2014) and performance outcomes (Fry et al., 2006; Keogh, Hume, Mellow, \& Pearson, 2005) of various strength based exercises including the bench press, clean and jerk, snatch and squat. Although Winwood, et al. (2012) investigated the correlations between a number of simple anthropometric measures to the performance of athletes undertaking strongman exercises in a simulated competition, no study to date has assessed how anthropometric measures influence the kinematics, kinetics or muscle activity patterns of any strongman exercise. Such studies have however been conducted 
665 for the snatch weightlifting event, whereby a number of anthropometric measures were found

666 to correlate to bar trajectory in elite female weightlifters (Musser, et al., 2014). Lower limb

667 length showed strong correlation to horizontal bar displacement during the first pull phase of

668 the snatch $(r=-0.93)$ in female $75 \mathrm{~kg}$ body mass class athletes, while thigh and lower limb

669 length showed strong correlation to horizontal bar displacement during the second pull phase

670 (thigh: $r=-0.99$, lower limb: $r=-0.94$ ) in female $53 \mathrm{~kg}$ body mass class athletes (Musser, et

671 al., 2014). Various body segment ratios also showed strong correlation to horizontal bar

672 displacement across the body mass classes (Musser, et al., 2014). The exploration of the effect

673 of anthropometrics (including limb lengths) on strongman biomechanics and the resultant

674 performance measure of an athlete may be particularly interesting in strongman exercises due

675 to the apparent variation in techniques used by strongman athletes of varying anthropometric

676 characteristics. These data may be used by strongman coaches when teaching and improving

677 the technique of an athlete so to enhance performance and prevent injury, while also being of

678 interest to strength and conditioning coaches who may wish to prescribe such exercises to their

679 non-strongman athletes. 


\section{Conclusion:}

681 The articles reviewed included the biomechanical analysis of eight different strongman exercises, with the farmers walk being the most commonly studied exercise as it appeared in

683 five of the ten studies. The majority of the articles reviewed were more applicable to strength 684 and conditioning coaches looking to implement strongman exercises into the training 685 programmes of non-strongman athletes than to strongman athletes and coaches looking to improve strongman competition performance. Although the population size and training experience of participants varied between the studies reviewed, all studies consisted of male participants of a largely similar lower level competitive standard, age and body mass. All studies reviewed were of a cross sectional observational study design and consisted of a warm up and testing component. The biomechanical measurements collected throughout the testing components could be categorised into six primary areas, however due to the general awkward nature of strongman exercises the methods used to collect biomechanical measurements were often constrained to 2D motion capture and/or force plate analysis.

It is recommended future research in the field of strongman biomechanics should: assess under/un-researched strongman exercises; include a greater number of experienced strongman athletes (including female and lighter weight males); compare biomechanical measures between strongman athletes of different performance standards; consider the collection of biomechanical data over a range of loading conditions (e.g. competition loads); utilise advanced measurement technologies (e.g. 3D and/or IMU motion capture) for the collection of data; consider how anthropometric measures (such as limb length) affect the biomechanics and performance of an athlete. With improvements in the research methodology of future strongman biomechanics studies, strength and conditioning coaches, and strongman athletes and coaches will be able to better understand; how strongman exercises may be used in wider strength and conditioning or injury rehabilitation practice, and the technique required 
705 to perform these exercises in a way that ensures the greatest performance outcome while 706 minimising the risk of injury.

707 
708 Acknowledgements:

709 The Authors wish to acknowledge Shiqi Thng for her contribution during the screening of

710 literature and quality/risk of bias assessment processes.

711

712

713 Funding details:

714 This research is supported by an Australian Government Research Training Program

715 Scholarship to the primary author.

716

717

718 Disclosure statement:

719 The Authors declare they have no conflicts of interest pertinent to the content of the review.

720

721 
723 Argus, C. K., Gill, N. D., Keogh, J. W. L., \& Hopkins, W. G. (2011). Assessing lower-body peak power in elite rugby-union players. Journal of Strength and Conditioning Research, 25(6), 1616-1621. doi: 10.1519/JSC.0b013e3181ddfabc

726

Ariëns, G. A. M., van Mechelen, W., Bongers, P. M., Bouter, L. M., \& van der Wal, G. (2000). Physical risk factors for neck pain. Scandinavian Journal of Work, Environment \& Health, 26(1), 7-19. doi: 10.5271/sjweh.504

Ball, N., \& Scurr, J. (2010). An assessment of the reliability and standardisation of tests used to elicit reference muscular actions for electromyographical normalisation. Journal of Electromyography and Kinesiology, 20(1), 81-88. doi: 10.1016/j.jelekin.2008.09.004

Berning, J. M., Adams, K. J., Climstein, M., \& Stamford, B. A. (2007). Metabolic demands of "junkyard" training: pushing and pulling a motor vehicle. Journal of Strength and Conditioning Research, 21(3), 853-856. doi: 10.1519/R-18335.1

Blair, S., Duthie, G., Robertson, S., Hopkins, W. G., \& Ball, K. (2018). Concurrent validation of an inertial measurement system to quantify kicking biomechanics in four football codes. Journal of Biomechanics, 73, 24-32. doi: 10.1016/j.jbiomech.2018.03.031

Blatnik, J. A., Goodman, C. L., Capps, C. R., Awelewa, O. O., Triplett, T. N., Erickson, T. M., \& McBride, J. M. (2014). Effect of load on peak power of the bar, body and system during the deadlift. Journal of Sports Science and Medicine, 13(3), 511-515. Retrieved from https://www.jssm.org

Chowdhury, R. H., Reaz, M. B., Ali, M. A., Bakar, A. A., Chellappan, K., \& Chang, T. G. (2013). Surface electromyography signal processing and classification techniques. Sensors, 13(9), 12431-12466. doi: $10.3390 / \mathrm{s} 130912431$

Cross, M. R., Tinwala, F., Lenetsky, S., Samozino, P., Brughelli, M., \& Morin, J. B. (2017). Determining friction and effective loading for sled sprinting. Journal of Sports Sciences, 35(22), 2198-2203. doi: $10.1080 / 02640414.2016 .1261178$

Davids, E. L., \& Roman, N. V. (2014). A systematic review of the relationship between parenting styles and children's physical activity. African Journal for Physical Health Education, Recreation and Dance, 20(Supplement 2), 228-246. Retrieved from https://journals.co.za/content/journal/ajpherd

DuRant, R. H. (1994). Checklist for the evaluation of research articles. Journal of Adolescent Health, 15(1), 4-8. doi: 10.1016/1054-139X(94)90381-6 
Escamilla, R. F., Francisco, A. C., Fleisig, G. S., Barrentine, S. W., Welch, C. M., Kayes, A. V., . . Andrews, J. R. (2000). A three-dimensional biomechanical analysis of sumo and conventional style deadlifts. Medicine and Science in Sports and Exercise, 32(7), 1265-1275. doi: 10.1097/00005768-20000700000013

Filippeschi, A., Schmitz, N., Miezal, M., Bleser, G., Ruffaldi, E., \& Stricker, D. (2017). Survey of motion tracking methods based on inertial sensors: a focus on upper limb human motion. Sensors, 17(6). doi: $10.3390 / \mathrm{s} 17061257$

Fry, A. C., Ciroslan, D., Fry, M. D., LeRoux, C. D., Schilling, B. K., \& Chiu, L. Z. (2006). Anthropometric and performance variables discriminating elite American junior men weightlifters. Journal of Strength and Conditioning Research, 20(4), 861-866. doi: 10.1519/R-18355.1

Gaviglio, C. M., Osborne, M., Kelly, V. G., Kilduff, L. P., \& Cook, C. J. (2015). Salivary testosterone and cortisol responses to four different rugby training exercise protocols. European Journal of Sport Science, 15(6), 497-504. doi: 10.1080/17461391.2015.1017012

Ghigiarelli, J. J., Sell, K. M., Raddock, J. M., \& Taveras, K. (2013). Effects of strongman training on salivary testosterone levels in a sample of trained men. Journal of Strength and Conditioning Research, 27(3), 738-747. doi: 10.1519/JSC.0b013e3182578115

Giroux, C., Rabita, G., Chollet, D., \& Guilhem, G. (2016). Optimal balance between force and velocity differs among world-class athletes. Journal of Applied Biomechanics, 32(1), 59-68. doi: 10.1123/jab.2015-0070

Guerra-Filho, G. (2005). Optical motion capture: theory and implementation. IEEE Revista Iberoamericana de $\begin{array}{llllll}\text { Tecnologias } & \text { del } & \text { Aprendizaje, } & \text { 12(2), } & \text { 61-90. } & \text { Retrieved }\end{array}$ https://ieeexplore.ieee.org/xpl/RecentIssue.jsp?punumber=6245520

Gyorkos, T. W., Tannenbaum, T. N., Abrahamowicz, M., Oxman, A. D., Scott, E. A., Millson, M. E., .. Best, A. (1994). An approach to the development of practice guidelines for community health interventions. Canadian Journal of Public Health, 85, S8-13. Retrieved from https://journal.cpha.ca/index.php/cjph

Hall, D. E., \& Moreland, J. C. (2001). Fundamentals of rolling resistance. Rubber Chemistry and Technology, 74(3), 525-539. doi: $10.5254 / 1.3547650$

Harris, N. K., Woulfe, C. J., Wood, M. R., Dulson, D. K., Gluchowski, A. K., \& Keogh, J. W. L. (2016). Acute physiological responses to strongman training compared to traditional strength training. Journal of Strength and Conditioning Research, 30(5), 1397-1408. doi: 10.1519/JSC.0000000000001217 

Company.

Keogh, J. W. L., Hume, P., Mellow, P., \& Pearson, S. (2005). The use of anthropometric variables to predict bench press and squat strength in well-trained strength athletes. International Symposium on Biomechanics in Sports: Conference Proceedings Archive, 126-129. Retrieved from https://ojs.ub.unikonstanz.de/cpa

Keogh, J. W. L., Kattan, A., Logan, S., Bensley, J., Muller, C., \& Powell, L. (2014). A preliminary kinematic gait analysis of a strongman event: the farmers walk. Sports, 2(1), 24-33. doi: 10.3390/sports2010024

Keogh, J. W. L., Newlands, C., Blewett, S., Payne, A. L., \& Chun-Er, L. (2010a). A kinematic analysis of a strongman-type event: the heavy sprint-style sled pull. Journal of Strength and Conditioning Research, 24(11), 3088-3097. doi: 10.1519/JSC.0b013e3181b62c2f

792

Keogh, J. W. L., Payne, A. L., Anderson, B. B., \& Atkins, P. J. (2010b). A brief description of the biomechanics and physiology of a strongman event: the tire flip. Journal of Strength and Conditioning Research, 24(5), 1223-1228. doi: 10.1519/JSC.0b013e3181cc61cd

Keogh, J. W. L., \& Winwood, P. W. (2017). The epidemiology of injuries across the weight-training sports. Sports Medicine, 47(3), 479-501. doi: 10.1007/s40279-016-0575-0

Ma, S., \& Zahalak, G. (1991). A distribution model of energetics in skeletal muslce. Journal of Biomechanics, 24(1), 21-35. doi: 10.1016/0021-9290(91)90323-f

800

801

802

McGill, S. M., \& Norman, R. W. (1985). Dynamically and statically determined low back moments during lifting. Clinical Biomechanics, 1(2), 116. doi: 10.1016/0268-0033(86)90125-7

McManus, A., O'Driscoll, J., Coleman, D. A., \& Wiles, J. (2016a). Strongman training (part 2): needs analysis 805 and integration into strength \& conditioning programming. Professional Strength \& Conditioning, 44, 7-15. Retrieved from https://www.uksca.org.uk/home

McManus, A., Wiles, J., Coleman, D. A., \& O'Driscoll, J. (2016b). Strongman training (part 1): a rationale for its inclusion in strength \& conditioning. Professional Strength \& Conditioning, 43, 7-18. Retrieved from https://www.uksca.org.uk/home 

barbell trajectory in the snatch lift for elite women weightlifters. Journal of Strength and Conditioning Research, 28(6), 1636-1648. doi: 10.1519/JSC.0000000000000450

813 Pearson, S. N., Cronin, J. B., Hume, P. A., \& Slyfield, D. (2009). Kinematics and kinetics of the bench-press and bench-pull exercises in a strength-trained sporting population. Sports Biomechanics, 8(3), 245-254. doi:

Picerno, P., Iannetta, D., Comotto, S., Donati, M., Pecoraro, F., Zok, M., . . Piacentini, M. F. (2016). 1RM prediction: a novel methodology based on the force-velocity and load-velocity relationships. European Journal of Applied Physiology, 116(10), 2035-2043. doi: 10.1007/s00421-016-3457-0

819 Rahmani, A., Viale, F., Dalleau, G., \& Lacour, J. R. (2001). Force/velocity and power/velocity relationships in squat exercise. European Journal of Applied Physiology, 84(3), 227-232. doi: 10.1007/PL00007956

Renals, L., Lake, J., Keogh, J. W. L., \& Austin, K. (2018). Strongman log push press: the effect log diameter has on force-time characteristics. Journal of Strength and Conditioning Research, 32(10), 2693-2700. doi: 10.1519/JSC.0000000000002764

Roman, N. V., \& Frantz, J. M. (2013). The prevalence of intimate partner violence in the family: a systematic review of the implications for adolescents in Africa. Family Practice, 30(3), 256-265. doi: 10.1093/fampra/cms084

Sanchez-Medina, L., Gonzalez-Badillo, J. J., Perez, C. E., \& Pallares, J. G. (2013). Velocity- and power-load

Schurr, S. A., Marshall, A. N., Resch, J. E., \& Saliba, S. A. (2017). Two-dimensional video analysis is comparable to 3D motion capture in lower extremity movement assessment. International Journal of Sports Physical Therapy, 12(2), 163-172. Retrieved from https://spts.org/member-benefits-detail/enjoy-memberbenefits/journals/ijspt

Shamseer, L., Moher, D., Clarke, M., Ghersi, D., Liberati, A., Petticrew, M., . . PRISMA-P. Group. (2015). Preferred reporting items for systematic review and meta-analysis protocols (PRISMA-P) 2015: elaboration and explanation. BMJ, 350, g7647. doi: 10.1136/bmj.g7647 vs. thigh muscles strength ratio and their relation to electromyography amplitude during a farmer's walk exercise. Journal of Human Kinetics, 45, 157-165. doi: 10.1515/hukin-2015-0016 

and hexagonal barbell deadlifts using submaximal loads. Journal of Strength and Conditioning Research, 25(7), 2000-2009. doi: 10.1519/JSC.0b013e3181e73f87

843 van der Windt, D. A., Thomas, E., Pope, D. P., de Winter, A. F., Macfarlane, G. J., Bouter, L. M., \& Silman, A. J. (2000). Occupational risk factors for shoulder pain: a systematic review. Occupational and Environmental Medicine, 57(7), 433-442. doi: 10.1136/oem.57.7.433

Vandenbroucke, J. P., von Elm, E., Altman, D. G., Gotzsche, P. C., Mulrow, C. D., Pocock, S. J., . . STROBE Initiative. (2007). Strengthening the reporting of observational studies in epidemiology (STROBE): explanation and elaboration. PLoS Med, 4(10), e297. doi: 10.1371/journal.pmed.0040297

849 Vigotsky, A. D., Halperin, I., Lehman, G. J., Trajano, G. S., \& Vieira, T. M. (2018). Interpreting signal amplitudes in surface electromyography studies in sport and rehabilitation sciences. Frontiers in Physiology, 8, 985. doi: 10.3389/fphys.2017.00985

Waller, M., Piper, T., \& Townsend, R. (2003). Strongman events and strength and conditioning programs. Strength and Conditioning Journal, 25(5), 44-52. doi: 10.1519/00126548-200310000-00008

Winwood, P. W., Cronin, J. B., Brown, S. R., \& Keogh, J. W. L. (2014a). A biomechanical analysis of the farmers walk, and comparison with the deadlift and unloaded walk. International Journal of Sports Science \& Coaching, 9(5), 1127-1143. doi: 10.1260/1747-9541.9.5.1127

Winwood, P. W., Cronin, J. B., Brown, S. R., \& Keogh, J. W. L. (2015a). A biomechanical analysis of the heavy

Winwood, P. W., Cronin, J. B., Brown, S. R., \& Keogh, J. W. L. (2015b). A biomechanical analysis of the strongman log lift and comparison with weightlifting's clean and jerk. International Journal of Sports Science \& Coaching, 10(5), 869-886. doi: 10.1260/1747-9541.10.5.869 sprint-style sled pull and comparison with the back squat. International Journal of Sports Science \& Coaching, 10(5), 851-868. doi: 10.1260/1747-9541.10.5.851 Strongman vs. traditional resistance training effects on muscular function and performance. Journal of Strength and Conditioning Research, 29(2), 429-439. doi: 10.1519/JSC.0000000000000629 
Winwood, P. W., Dudson, M. K., Wilson, D., McLaren-Harrison, J. K. H., Redjkins, V., Pritchard, H. J., \& Keogh, J. W. L. (2018). Tapering practices of strongman athletes. Journal of Strength and Conditioning Research, 32(5), 1181-1196. doi: 10.1519/JSC.0000000000002453

872 Winwood, P. W., Hume, P. A., Cronin, J. B., \& Keogh, J. W. L. (2014c). Retrospective injury epidemiology of strongman athletes. Journal of Strength and Conditioning Research, 28(1), 28-42. doi: 10.1519/JSC.0b013e3182986c0c

Winwood, P. W., Keogh, J. W. L., \& Harris, N. K. (2011). The strength and conditioning practices of strongman competitors. Journal of Strength and Conditioning Research, 25(11), 3118-3128. doi: 10.1519/JSC.0b013e318212daea

878

Winwood, P. W., Keogh, J. W. L., \& Harris, N. K. (2012). Interrelationships between strength, anthropometrics, and strongman performance in novice strongman athletes. Journal of Strength and Conditioning

Wong, W. C., Cheung, C. S., \& Hart, G. J. (2008). Development of a quality assessment tool for systematic reviews of observational studies (QATSO) of HIV prevalence in men having sex with men and associated risk behaviours. Emerging Themes in Epidemiology, 5, 23. doi: 10.1186/1742-7622-5-23

Woulfe, C. J., Harris, N. K., Keogh, J. W. L., \& Wood, M. R. (2014). The physiology of strongman training. Strength and Conditioning Journal, 36(6), 84-95. doi: 10.1519/Ssc.0000000000000104

Zemke, B., \& Wright, G. (2011). The use of strongman type implements and training to increase sport performance in collegiate athletes. Strength and Conditioning Journal, 33(4), 1-7. doi: 